\title{
Bewegung im Datenschutz
}

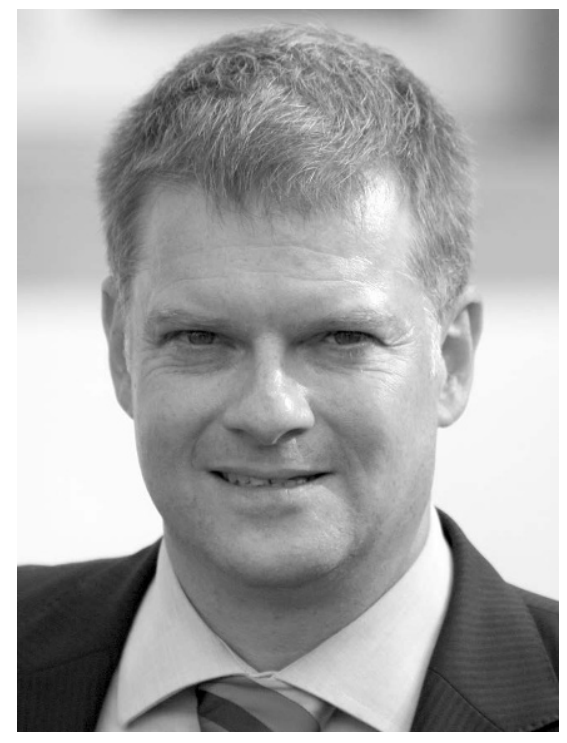

Wenn die Datenschutz-Grundverordnung im ersten Jahr ihrer Geltung etwas bewirkt hat, dann ist es vor allem dies: Sie hat Bewegung in zahlreiche wichtige Datenschutzfragen gebracht.

So könnte die bevorstehende Entscheidung des Europäischen Gerichtshofs über die Klage gegen die Einbindung von Facebook-Like-Buttons im Kontext der "gemeinsamen Verantwortlichkeit" neue Pflichten für Webseitenbetreiber begründen, wie Gerhard Kunnert in seiner Analyse der Schlussanträge des Generalanwalts ausführt.

Auch die deutliche Ausrichtung der Grundverordnung an der Nachweisbarkeit von Datenschutz-Vorgängen - wie Einwilligungen, Widerrufen, Vorfällen, Unterweisungen etc. - ruft nach neuen Antworten: Ohne ein Management-System, wie wir es in der Informationssicherheit schon lange aus dem ISO/IEC-Standard 27001 kennen, wird der Datenschutz diese Anforderungen auf mittlere Sicht kaum erfüllen können. Dominik Schrahe und Thomas Städter diskutieren in ihrem Beitrag, ob und wie sich beide Management-Systeme integrieren lassen.

Eine sehr interessante Untersuchung zur Wirkung der Transparenzpflichten steuern Tim Zander, Anne Steinbrück und Pascal Birnstill bei: Mit Methoden der Spieltheorie analysieren sie deren ausgleichende Wirkung auf die Informationsasymmetrie zwischen Anbieter und Betroffenen.

Dass die datenschutzrechtlichen Informationspflichten in der Praxis nicht notwendig zu adäquatem Nutzerverständnis (und entsprechendem Verhalten) führen, weisen Jacqueline Brendel und Nina Gerber in ihrer Untersuchung von Messenger-Diensten nach: Nicht nur werden das Konzept der Ende-zu-Ende-Verschlüsselung und dessen technische Grenzen (Schutz von Meta-Daten) von Anwendern häufig nicht verstanden, sondern sind auch die Verschlüsselungsfunktionen in den Messengern oft so integriert, dass es zu Fehlverständnissen bei deren Benutzung kommt.

Der Frage der Authentizität und Integrität von Bildern (angesichts immer besseren Bildbearbeitungsverfahren) und deren Nachweisbarkeit mit Hilfe kryptografischer Verfahren und der Nutzung von Trusted Platform Modules gehen Eckehard Hermann, Harald Lampesberger, Lena Heimberger, und Michael Altenhuber in ihrem Beitrag nach. Anschließend stellt Stefan Beißel Methoden zur Evaluation von Security-Awareness-Maßnahmen vor, die sich auch auf Datenschutz-Awareness-Maßnahmen anwenden lassen.

Schließlich zeichnen René Thiele und Gerhard Kramarz - von Kohout die voraussichtlichen Auswirkungen der bis 2020 in nationales Recht umzusetzenden „Richtlinie über den europäischen Kodex für die elektronische Kommunikation“ für ein modernes, barrierefreies und auf private Netze erweitertes Notrufregime voraus.

Eine anregende Lektüre wünscht Ihnen

\section{Dirk Fox}

